\title{
Which Linear Compartmental Systems Contain Traps?
}

DANIEL FIFE

University of Michigan Medical School, Ann Arbor, Michigan, 48104

Communicated by John Jacquez

This paper will prove the following result which was stated without proof by Dr. John Jacquez: A linear compartmental system has a trap if and only if the associated system of differential equations has a zero eigenvalue. It will then use this result to prove an approximation theorem which says roughly that a linear compartmental system has an approximate trap if and only if the associated system of differential equations has an eigenvalue which is approximately zero.

Let $S$ represent a linear compartmental system consisting of compartments $C_{1}, C_{2}, \ldots C_{n}$ and $q_{j}$ be the amount of material in $C_{j}$. Let $f_{i, j}$ be the fractional exchange coefficient so that the rate of flow of material from $C_{j}$ to $C_{i}$ is $f_{i, j} q_{j}$; and let $f_{0, j} q_{j}$ be the rate of flow of material from $C_{j}$ to the environment. The total outflow from $C_{j}$ is $\left(f_{0, j}+\Sigma_{i \neq j} f_{i, j}\right) q_{j}$ which we will write as $f_{j, j} q_{j}$. This leads us to consider the system of differential equations,

$$
\dot{q}_{j}=\Sigma^{\prime} f_{j, i} q_{i}-f_{j, j} q_{j}, \quad j=1 \ldots n,
$$

where we write $\Sigma^{\prime}$ for $\Sigma_{i \neq j}$. Equation (1) may be written

$$
\dot{q}=F q,
$$

where $q$ is the column vector whose entries are $q_{1}, \ldots, q_{n}$ and $F$ is the matrix given by

$$
F_{i, j}=\left\{\begin{aligned}
f_{i, j} & \text { if } i \neq j \\
-f_{j, j} & \text { if } i=j .
\end{aligned}\right.
$$

$\lambda$ is an eigenvalue of Eq. 2 just if $\operatorname{det}(F-\lambda I)=0$. Thus $\lambda=0$ is an eigenvalue just if $\operatorname{det}(F)=0$.

What do we mean by a trap? We mean a subsystem with no output (to things outside itself). Suppose $T \subseteq S$ and renumbering compartments need be, $T=C_{m}, \ldots, C_{n}(m \leqslant n)$. $T$ is a trap if and only if $f_{i, j}=0$ for all $(i, j)$ such that $j \geqslant m$ and $i<m$ (including $i=0$ ).

In stating the above it was convenient to renumber the compartments of $S$. What does this do to the matrix $F$ ? Renumbering amounts to applying some permutation $P$ to the subscripts of $C_{1}, \ldots, C_{n}$. The new matrix 
representing the new system is obtained by applying $P$ to both the rows and the columns of the old matrix [1]. This is easy to see in case $P$ merely switches the names of two compartments. Since any permutation can be written as a series of switches the result follows.

\section{THEOREM 1}

$S$ has a trap if and only if either:

(a) each column of F sums to zero or,

(b) There is a permutation which can be applied to the rows and columns of $F$ to give a matrix of the form $\left(\begin{array}{ll}U & 0 \\ Q & R\end{array}\right)$ where 0 consists only of zeros, $U$ and $R$ are square and each column of $R$ sums to zero.

" $a$ " corresponds to the case $T=S$ and " $b$ " to the case $T \subset S$.

\section{Proof}

The following are all equivalent.

i. $T \subseteq S$ is a trap (and may be written $T=C_{m}, \ldots, C_{n}$ )

ii. $f_{i, j}=0$ for all $(i, j)$ such that $j=m, \ldots, n$ and $i=0, \ldots, m-1$.

iii. $F_{i, j}=0$ for all $(i, j)$ such that $j=m, \ldots, n$ and $i=1, \ldots, m-1$ and (Eq. 3 plus $f_{0, j}=0$ ) $F_{j, j}=-\Sigma_{i} F_{i, j}, j=m, \ldots, n$.

iv. Statement "b" if $m>1$ or statement "a" if $m=1$.

\section{THEOREM 2}

$S$ has a trap if and only if zero is an eigenvalue of Eq. (2).

\section{Proof}

Recall that zero is an eigenvalue of Eq. 2 if and only if $\operatorname{det}(F)=0$. Suppose $S$ has a trap $T$. If $T=S$ then from theorem 1 each column of $F$ sums to zero so $\operatorname{det}(F)=0$. If $T \subset S$ permute $F$ to get it in the form mentioned in theorem 1 part b. Each column of $R$ sums to zero so $\operatorname{det}(R)=0$. Hence the columns of $R$ are linearly dependent. So are the columns of $F$ which pass through $R$ since 0 consists of zeros only. Hence $\operatorname{det}(F)=0$.

Suppose $\operatorname{det}(F)=0$. From Eq. 3 and the definition of $f_{i, j}$ we see that $\Sigma_{i} F_{i, j}=0$ just if $\left|F_{j, j}\right|=\Sigma_{i}^{\prime}\left|F_{i, j}\right|$. Finally, since $f_{i \cdot j} \geqslant 0,\left|F_{j, j}\right| \geqslant \Sigma_{i}^{\prime}\left|F_{i, j}\right|$. We will show that a matrix with the above three properties must satisfy " $a$ " or " $b$ " of theorem 1 and this will complete the proof. The lemma is a restatement of a result given by $O$. Tausky [2].

Let $\boldsymbol{A}=\left(a_{i, j}\right)$ be an $n \times n$ real or complex matrix and $A_{j}=\Sigma_{i}^{\prime}\left|a_{i, j}\right|$. Let $x=\left(x_{1}, \ldots, x_{n}\right)$. We will say $\left|x_{j}\right|$ is maximal if $\left|x_{j}\right| \geqslant\left|x_{i}\right|$, all $i \neq j$. 


\section{$L E M M A$}

Suppose

(1) $\operatorname{Det}(A)=0$ and

(2) $\left|a_{j, j}\right| \geqslant A_{j}$ all $j$.

Then either

I. $\left|a_{i, i}\right|=A_{i}$ for all $i$ or,

II. A can be transformed to the form $\left(\begin{array}{ll}U & 0 \\ Q & R\end{array}\right)$ by the same permutation of its rows and columns where $U$ and $R$ are square matrices and 0 consists entirely of zeros. For those columns $t$ which fall in $R$ we also have $\left|a_{t, t}\right|=A_{t}$.

Recall that II is equivalent to $b$ of theorem 1 because of the second property mentioned above.

\section{Proof of lemma}

Since $\operatorname{det}(A)=0$ there is a nonzero $X=\left(x_{1}, \ldots, x_{n}\right)$ solving $X A=0$. We may permute the rows of $A$ (and the columns of $A$ the same way) so that $\left|x_{1}\right| \leqslant\left|x_{2}\right| \leqslant \ldots \leqslant\left|x_{n}\right|$. Suppose all $\left|x_{i}\right|$ are maximal. From the $i$ th equation of $X A=0$ we have

$$
\begin{aligned}
& -x_{i} a_{i, i}=\Sigma_{j}^{\prime} x_{j} a_{j, i}, \\
& \left|x_{i}\right|\left|a_{i, i}\right| \leqslant \Sigma_{j}^{\prime}\left|x_{j}\right|\left|a_{j, t}\right|,
\end{aligned}
$$

and since all $\left|x_{j}\right|$ are equal

$$
\left|a_{i, i}\right| \leqslant \Sigma^{\prime}\left|a_{j, i}\right|=A_{i} .
$$

With " 2 " this gives $\left|a_{i, i}\right|=A_{i}$ (for all $i$ ).

The other possibility is that (at least) $\left|x_{1}\right|<\left|x_{n}\right|$. Let $m$ be the lowest index for which $\left|x_{m}\right|=\left|x_{n}\right|$. If $t \geqslant m$ we see as we did before that

$$
\left|x_{t}\right|\left|a_{t, t}\right| \leqslant \Sigma_{j}\left|x_{j}\right|\left|a_{j, t}\right| \text {. }
$$

Hence, $\left|a_{t, t}\right|<\Sigma_{j}^{\prime}\left|a_{j, t}\right|$ (contradicting 2) unless all the $a_{i, t}$ for which $\left|x_{i}\right|<\left|x_{t}\right|$ are zero. Thus $a_{i, t}=0, i=1, \ldots, m-1$ and this is true for all $t=m, \ldots, n$. This gives the required block of zeros.

If column $t$ falls in $R$ (i.e. if $t \geqslant m$ ) then Eq. 7 applies. The first $n-1$ terms in the sum are zero. For the rest $\left|x_{j}\right|=\left|x_{t}\right|$ so $\Sigma_{j}^{\prime}\left|a_{j, t}\right| \geqslant\left|a_{t, t}\right|$ and as before this gives $A_{\mathrm{t}}=\left|a_{t, t}\right|$.

We will now use the above theorem to prove an approximate result which will say that under broad conditions $\operatorname{det}(F)$ is approximately zero if and only if $F$ has an eigenvalue which is approximately zero and this happens if and only if $S$ has a subsystem which is approximately a trap. In order to state and prove such a result we will need to introduce some additional notation.

For a given set $C_{1}, \ldots, C_{n}$ we can consider $\mathscr{P}$, the set of all compartmental systems on $C_{1}, \ldots, C_{n}$, and $\mathscr{F}$, the set of all matrices of such 
systems. Since a choice of particular system $S \in \mathscr{P}$ is equivalent to a choice of a set of fractional transfer coefficients there is an obvious one to one correspondence between $\mathscr{S}$ and $\mathscr{F}$. In $\mathscr{F}$ we will say as usual that a sequence of matrices $F_{j}$ converges to a given matrix $F_{0}\left(F_{j} \rightarrow F_{0}\right)$ if and only if each entry of $F_{j}$ converges to the corresponding entry of $F_{0}$. The determinant is then a continuous function from $\mathscr{F}$ to the real numbers $R$.

$S \in \mathscr{S}$ has an approximate trap if it has a subsystem whose total output is small. More formally let $T \subseteq\left\{C_{1}, \ldots, C_{n}\right\}$. Without loss of generality $T=\left\{C_{m}, \ldots, C_{n}\right\}$. Then for any $S \in \mathscr{S}$ we define

$$
L_{T}(S)=\sum_{\substack{i<m \\ j \geqslant m}} f_{i, j}
$$

so that $L_{T}$ is the sum of the fractional coefficients for transfer out of $T$. We have the following picture

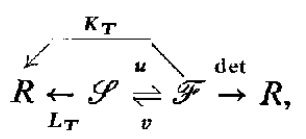

where $u$ and $v$ give the correspondence between $\mathscr{S}$ and $\mathscr{F}$ and $K_{T}=L_{T} \cdot v$. $K_{T}$ is a continuous function from $\mathscr{F}$ to $R$ for each $T$.

Let $K(F)=\min _{T} K_{T}(F)$ taking the minimum over all nonempty $T \subseteq\left\{C_{1}, \ldots, C_{n}\right\} . K$ is a continuous function from $\mathscr{F}$ to $R$. We have the picture

$$
R \underset{K}{\stackrel{\mathscr{S}}{\stackrel{\mathscr{F}}{\mathscr{F}} \underset{\operatorname{det}}{\rightarrow}} R}
$$

By Theorem 2, $K(F)=0$ if an only if $\operatorname{det}(F)=0$.

\section{THEOREM 3}

Suppose $\left\{F_{j}\right\}$ is a sequence of matrices in $\mathscr{F}$ and $F_{j} \rightarrow F_{0}$. Then $\operatorname{det}\left(F_{j}\right)$ $\rightarrow 0$ if and only if $K\left(F_{j}\right) \rightarrow 0$.

\section{Proof}

$\operatorname{det}\left(F_{j}\right) \rightarrow \operatorname{det}\left(F_{0}\right)$ so $\operatorname{det}\left(F_{j}\right) \rightarrow 0$ if and only if $\operatorname{det}\left(F_{0}\right)=0$. Similarly $K\left(F_{j}\right) \rightarrow 0$ if and only if $K\left(F_{0}\right)=0 . K\left(F_{0}\right)=0$ if and only if $\operatorname{det}\left(F_{0}\right)=0$.

A set of matrices is said to be bounded if the set of all the entries in all the matrices is a bounded set of numbers. It is a standard result that any closed bounded set of $n \times n$ matrices is compact.

\section{THEOREM 4}

Suppose $\left\{F_{j}\right\}$ is a bounded sequence of matrices in $\mathscr{F}$. Then $\operatorname{det}\left(F_{j}\right) \rightarrow 0$ if and only if $K\left(F_{j}\right) \rightarrow 0$. 
Proof

Suppose the contrary. By compactness we can choose a convergent subsequence with the same property. This contradicts theorem 3 .

Finally, we should point out that for $F$ bounded, $\operatorname{det}(F)$ is approximately zero if and only if $F$ has an eigenvalue which is approximately zero. To see this let $\Lambda_{j}$ be the set of eigenvalues of $F_{j}$. The product of all the $\lambda$ 's in $\Lambda_{j}$ is $\operatorname{det}\left(F_{j}\right)$ so at least one $\lambda$ in $\Lambda_{j}$ is no bigger than $\left|\operatorname{det}\left(F_{j}\right)\right|^{1 / n}$. On the other hand if $M$ is the bound on the entries of the matrices it is easy to see that each $\lambda$ in $\Lambda_{j}$ must satisfy $|\lambda| \leqslant n M$. If $\lambda$ is in $\Lambda_{j}$ we have $\left|\operatorname{det}\left(F_{j}\right)\right| \leqslant$ $(n M)^{n-1} \lambda$. Thus $\operatorname{det}\left(F_{j}\right) \rightarrow 0$ if and only if $\min \left\{|\lambda|\right.$ such that $\left.i \in \Lambda_{j}\right\} \rightarrow 0$.

This is the result we were after except for the requirement that the sequence be bounded. The following example shows that the boundedness condition cannot simply be dropped.

Let $S_{j}$ be given by

$$
\begin{gathered}
F_{j}=\left(\begin{array}{rr}
-j & 1 / j \\
0 & -1 / j
\end{array}\right) \\
C_{2} \stackrel{i, j}{\rightarrow} C_{1} \stackrel{j}{\rightarrow} \\
K\left(F_{j}\right)=\min (1 / j, j)=1 / j, \text { so } K\left(F_{j}\right) \rightarrow 0 .
\end{gathered}
$$

On the other hand $\operatorname{det}\left(F_{j}\right)=1$ for all $j$.

My attempts to construct an example in which $K\left(F_{j}\right)$ stayed away from zero and $\operatorname{det}\left(F_{j}\right) \rightarrow 0$ were frustrated. The core of the trouble was that according to theorem 4 any such sequence must be unbounded. However, if we notice that in evaluating $\operatorname{det}\left(F_{j}\right)$ all terms have the same sign and if we notice that $K\left(F_{j}\right)$ is a minimum of several sums of positive terms, it becomes clear that any unbounded sequence in which $K\left(F_{j}\right)$ stays away from zero and $\operatorname{det}\left(F_{j}\right) \rightarrow 0$ can be replaced by a bounded sequence with the same properties. (The terms which grow unboundedly can simply be replaced by suitable nonzero constants.) Thus no such example exists. The reader who prefers ending on a positive note may restate this as a theorem.

This work was supported by Grant 5TI GM 892-09 from the National Institutes of Health, USPHS.

\section{REFERENCES}

1 J. Z. Hearon, N.Y. Acad. Sci. Ann. 108, 36 (1963).

2 O. Tausky, Am. Math. Monthly 56, 10, 672 (1949). 